\title{
Positive Scheme Numerical Simulation of High Mach Number Astrophysical Jets
}

\author{
Youngsoo Ha • Carl L. Gardner
}

Received: 17 April 2007 / Revised: 21 September 2007 / Accepted: 4 October 2007 /

Published online: 27 October 2007

(C) Springer Science+Business Media, LLC 2007

\begin{abstract}
High Mach number astrophysical jets are simulated using a positive scheme, and are compared with WENO-LF simulations. A version of the positive scheme has allowed us to simulate astrophysical jets with radiative cooling up to Mach number 270 with respect to the heavy jet gas, a factor of two times higher than the maximum Mach number attained with the WENO schemes and ten times higher than with CLAWPACK. Such high Mach numbers occur in many settings in astrophysical flows, so it is important to develop a scheme that can simulate at these Mach numbers.
\end{abstract}

Keywords Astrophysical jets $\cdot$ Radiative cooling $\cdot$ Positive scheme simulations

\section{Introduction}

Images obtained with the Hubble Space Telescope have revealed intricate details in high Mach number gas dynamical flows of astrophysical jets [1], including shock wave patterns and Kelvin-Helmholtz vortex rollup. Numerical simulations [2, 3] at high Mach numbers (up to 80) with radiative cooling have been performed to provide physical insights into these flows (the usual convention is to specify the Mach number of the jet with respect to the jet gas).

In the astrophysical setting, the jet gas is on the order of ten times the density of the ambient gas, so we are interested here in simulating heavy jets (which are nearly "ballistic") rather than light jets (which have contact instabilities and a great deal of entrainment of the

Research supported in part by the BK21 project at KAIST.

Research supported in part by the Space Telescope Science Institute under grant HST-GO-09863.06-A.

Y. Ha

Division of Applied Mathematics, Korean Advanced Institute of Science and Technology,

305-701 Taejon, South Korea

e-mail: youngsooh@kaist.ac.kr

C.L. Gardner $(\varangle)$

Department of Mathematics and Statistics, Arizona State University, 85287-1804 Tempe, AZ, USA

e-mail: gardner@math.asu.edu 
ambient gas into the jet gas). Simulations at high Mach numbers and with radiative cooling are essential for achieving detailed agreement with the astrophysical observations. The gas flows in the HH 1-2 astrophysical jets [1] are at about Mach 80, and other astrophysical jets are at even higher Mach numbers.

In this investigation, we simulate the evolution of high Mach number astrophysical jets with radiative cooling using a positive scheme introduced by Liu and Lax [4, 5], and compare with results [2] obtained with the third- and fifth-order WENO-LF methods [6, 7]. Robust numerical methods are needed to handle the intense radiative shocks in front of (bow shock) and within the propagating jet (including the terminal Mach disk ${ }^{1}$ ), as well as the Kelvin-Helmholtz vortex rollup of the jet tip. Note that simulations with radiative cooling are more difficult to perform, because radiative shocks are stronger than shocks without cooling. High-order hyperbolic numerical methods developed over the last few decades perform well up to medium Mach numbers, but eventually produce negative pressures or densities as the Mach number of the jet is increased: CLAWPACK $[8,9]$ with the HLLE Riemann solver and component-wise limiting (supplied by LeVeque) can simulate the jets up to Mach 25 with radiative cooling, but then fails due to the generation of negative pressures in the Riemann solver around the rollup of the jet tip; WENO-LF can simulate the jets up to Mach 135 with radiative cooling. Positive schemes allow us to simulate astrophysical jets with radiative cooling at very high Mach numbers, up to Mach 270—much higher than other methods.

A conservative finite difference/volume method for a hyperbolic system of conservation laws is positive $[4,5]$ if it can be written in the form

$$
q_{J}^{n+1}=\sum_{K} C_{K} q_{J+K}^{n}
$$

where the coefficient matrices $C_{K}=C_{K}\left(q_{J}^{n}\right)$ have the following properties: (i) each $C_{K}$ is symmetric positive definite, (ii) $\sum_{K} C_{K}$ is the identity matrix, and (iii) $C_{K}=0$ except for a finite set of $K$. The positive scheme outlined below is able to simulate jets with radiative cooling at much higher Mach numbers than other schemes. We will present simulations of high Mach number astrophysical jets without and with radiative cooling using this positive scheme, and compare with WENO-LF simulations.

\section{Gas Dynamical Equations with Radiative Cooling}

The Euler equations of gas dynamics (in two dimensions) consist of equations for conservation of mass, momentum, and energy:

$$
\begin{aligned}
& \frac{\partial \rho}{\partial t}+\frac{\partial}{\partial x}(\rho u)+\frac{\partial}{\partial y}(\rho v)=0, \\
& \frac{\partial}{\partial t}(\rho u)+\frac{\partial}{\partial x}\left(\rho u^{2}+P\right)+\frac{\partial}{\partial y}(\rho u v)=0, \\
& \frac{\partial}{\partial t}(\rho v)+\frac{\partial}{\partial x}(\rho u v)+\frac{\partial}{\partial y}\left(\rho v^{2}+P\right)=0,
\end{aligned}
$$

\footnotetext{
${ }^{1}$ As the fast jet gas is slowed down to match the slower (but still supersonic) speed of the jet tip contact, a terminal Mach disk shock is formed.
} 


$$
\frac{\partial E}{\partial t}+\frac{\partial}{\partial x}(u(E+P))+\frac{\partial}{\partial y}(v(E+P))=-n^{2} \Lambda(T),
$$

where $\rho=m_{H} n$ is the density of the gas (predominantly $\mathrm{H}$ ), $m_{H}=938.272 \mathrm{MeV} / c^{2}$ is the mass of $\mathrm{H}, n$ is the number density, $(u, v)$ is the velocity, $\rho(u, v)$ is the momentum density, $P=n k_{B} T$ is the pressure, $k_{B}$ is Boltzmann's constant, $T$ is the temperature, and

$$
E=\frac{3}{2} n k_{B} T+\frac{1}{2} \rho\left(u^{2}+v^{2}\right)
$$

is the energy density. The pressure is related to the internal energy density by the equation of state, which to an excellent approximation is polytropic:

$$
P=(\gamma-1)\left(E-\frac{1}{2} \rho\left(u^{2}+v^{2}\right)\right),
$$

where the polytropic gas constant $\gamma=5 / 3$ for a monatomic gas like $\mathrm{H}$.

Radiative cooling of the gas is incorporated through the right-hand side of (5), with the model for $\Lambda(T)$ taken from Fig. 8 of [10]. The cooling law can be modeled approximately by

$$
\left(\frac{d E}{d t}\right)_{\text {cooling }}=-n^{2} \Lambda(T) \approx \begin{cases}-\tilde{\Lambda}\left(P^{2}-P_{a}^{2}\right), & T>T_{a}, \\ 0, & \text { otherwise, }\end{cases}
$$

where $\tilde{\Lambda}=8.776$ in our computational units, $P_{a}$ is the ambient pressure, and $T_{a}$ is the ambient temperature.

\section{Numerical Method}

We will use a positive scheme for jet flows with radiative cooling and for Mach numbers up to 270 .

The radiative cooling source term in the Euler equations can be handled via either a split or unsplit method-here we use a split method: first we solve the homogeneous gas dynamics equations (with $\Lambda \equiv 0$ ), and then we update the energy density $E$ by solving the ordinary differential equation

$$
\left(\frac{d E}{d t}\right)_{\text {cooling }}=-n^{2} \Lambda(T)
$$

\subsection{Conservation Laws}

To describe the positive scheme, we consider a general system of hyperbolic conservation laws:

$$
\frac{\partial q}{\partial t}+\frac{\partial f(q)}{\partial x}+\frac{\partial g(q)}{\partial y}=0,
$$

where $q=\left[q_{1}, q_{2}, \ldots, q_{m}\right]^{T}, f=\left[f_{1}, f_{2}, \ldots, f_{m}\right]^{T}$, and $g=\left[g_{1}, g_{2}, \ldots, g_{m}\right]^{T}$. Here $q$ is the vector of conserved variables, and $f=f(q)$ and $g=g(q)$ are the vector fluxes.

Equation (10) can be written in the quasi-linear form

$$
\frac{\partial q}{\partial t}+A(q) \frac{\partial q}{\partial x}+B(q) \frac{\partial q}{\partial y}=0
$$


where $A(q)=f^{\prime}(q)$ and $B(q)=g^{\prime}(q)$ are the symmetrizable flux Jacobian matrices.

Discretize the $x$ and $y$ axes with mesh widths $\Delta x$ and $\Delta y$, respectively, and define the midpoints

$$
x_{j+1 / 2}=x_{j}+\frac{\Delta x}{2}, \quad y_{k+1 / 2}=y_{k}+\frac{\Delta y}{2} .
$$

Next define cell averages of $q\left(x_{j}, y_{k}, t^{n}\right)$ by

$$
\bar{q}_{j, k}^{n} \equiv \frac{1}{\Delta x \Delta y} \int_{x_{j-1 / 2}}^{x_{j+1 / 2}} d x \int_{y_{k-1 / 2}}^{y_{k+1 / 2}} d y q\left(x, y, t^{n}\right)
$$

based on spatial cells $I_{j, k}=\left[x_{j-1 / 2}, x_{j+1 / 2}\right] \times\left[y_{k-1 / 2}, y_{k+1 / 2}\right]$. The conservation laws (10) in semi-discrete form are

$$
\frac{d}{d t} \bar{q}_{j, k}(t)=-\frac{\hat{f}_{j+\frac{1}{2}, k}(t)-\hat{f}_{j-\frac{1}{2}, k}(t)}{\Delta x}-\frac{\hat{g}_{j, k+\frac{1}{2}}(t)-\hat{g}_{j, k-\frac{1}{2}}(t)}{\Delta y} \equiv \mathcal{L}\left(\bar{q}^{n} ; j, k\right),
$$

where $\hat{f}_{j+\frac{1}{2}, k}$ and $\hat{g}_{j, k+\frac{1}{2}}$ are numerical flux functions which are Lipschitz continuous in all arguments and consistent with the physical flux functions $f$ and $g$, respectively.

Then the solution to the conservative scheme will converge to a weak solution of the conservation laws (10), if it exists, by the Lax-Wendroff theorem [11].

\subsection{Positive Schemes}

The basic idea of the positive schemes $[4,5]$ is to combine a second-order accurate scheme with numerical flux $f^{a c c}$ with a dissipative scheme with numerical flux $f^{\text {diss }}$. Then using the flux limiter introduced in $[12,13]$, the total numerical flux is of the form

$$
\hat{f}=f^{\text {diss }}+L\left(f^{a c c}-f^{d i s s}\right),
$$

where the flux limiter $L$ is near the identity when the flow is smooth and near zero otherwise. In this way the positive scheme attains high-order accuracy in regions of smooth flow, and sharp monotone resolution of shock waves.

For two-dimensional hyperbolic conservation laws, we use a dimension-by-dimension spatial splitting. The numerical flux function $\hat{f}_{j+1 / 2}$ for the $x$ sweep (we omit the index $k$ for the $y$ direction) is given by

$$
\hat{f}_{j+1 / 2}=\frac{f\left(q_{j}\right)+f\left(q_{j+1}\right)}{2}-\frac{1}{2} R[\alpha|\Lambda|(I-\Phi)+\beta \mu(I-\Psi)] R^{-1}\left(q_{j+1}-q_{j}\right),
$$

where the matrices $R,|\Lambda|, \Phi, \mu$, and $\Psi$ are defined below, and the adjustable parameters $\alpha$ and $\beta$ satisfy $0 \leq \alpha \leq 1$ and $\alpha+\beta \geq 1$. This positive scheme makes use of Roe's scheme [14], with an "entropy fix" [15] which guarantees a nonzero diffusive term through the choice of $\mu$ and $\beta .^{2}$ An advantage—and a disadvantage — of positive schemes is that they have many parameters which can be adjusted to obtain high resolution of different flows.

\footnotetext{
${ }^{2}$ Specifically, if $\lambda_{i}=0$, a nonzero diffusive term is constructed by choosing $\mu_{i}$ and $\beta$ to be positive.
} 
Characteristic decomposition proceeds in the following way: based on the values of $q_{j}$ and $q_{j+1}$, a mean Jacobian $A_{j+1 / 2}$ is defined at the interface $x_{j+1 / 2}$. We use Roe averaging [14] to compute the fluxes (16). Roe's method between states $q_{j}$ and $q_{j+1}$ is based on the averaged states

$$
\begin{aligned}
& \bar{u}=\frac{\sqrt{\rho_{j}} u_{j}+\sqrt{\rho_{j+1}} u_{j+1}}{\sqrt{\rho_{j}}+\sqrt{\rho_{j+1}}}, \\
& \bar{v}=\frac{\sqrt{\rho_{j}} v_{j}+\sqrt{\rho_{j+1}} v_{j+1}}{\sqrt{\rho_{j}}+\sqrt{\rho_{j+1}}}, \\
& \bar{H}=\frac{\sqrt{\rho_{j}} H_{j}+\sqrt{\rho_{j+1}} H_{j+1}}{\sqrt{\rho_{j}}+\sqrt{\rho_{j+1}}},
\end{aligned}
$$

where the total enthalpy $H=(E+p) / \rho$. The Roe averaging matrix is simply the Jacobian matrix $f^{\prime}(\bar{q})$ based on these averaged states. Write $A=R \Lambda R^{-1}$, where $\Lambda$ is the matrix of eigenvalues of $A$ and $R$ consists of columns of the right eigenvectors associated with the eigenvalues. $|\Lambda|=\operatorname{diag}\left\{\left|\lambda_{i}\right|\right\}, i=1, \ldots, m$, is a diagonal matrix whose entries are the absolute values of the eigenvalues $\lambda_{i}$ of $A$. Each entry of the diagonal matrix $\operatorname{diag}\left\{\mu_{i}\right\}$ satisfies $\mu_{i} \geq\left|\lambda_{i}\right|$. The matrices $\Phi=\operatorname{diag}\left\{\phi_{i}\left(\theta_{i}\right)\right\}$ and $\Psi=\operatorname{diag}\left\{\psi\left(\theta_{i}\right)\right\}$ have entries which are the limiter functions evaluated at the local solution, where $\theta_{i}$ is the ratio of the slope at the interface in the upwind direction to the slope at the current interface:

$$
\theta_{i}=\frac{l_{i}\left(q_{J+1}-q_{J}\right)}{l_{i}\left(q_{j+1}-q_{j}\right)}, \quad J= \begin{cases}j-1, & \text { if } \lambda_{i} \geq 0, \\ j+1, & \text { otherwise }\end{cases}
$$

for $i=1, \ldots, m$, and where $l_{i}$ is the $i$ th row of $R^{-1}$.

We use limiter functions [16] satisfying the constraints

$$
0 \leq \phi_{i}(\theta) \leq 2, \quad 0 \leq \frac{\phi_{i}(\theta)}{\theta} \leq 2, \quad \phi_{i}(1)=1
$$

and

$$
0 \leq \psi(\theta) \leq 1, \quad 0 \leq \frac{\psi(\theta)}{\theta} \leq 1, \quad \psi(1)=1 .
$$

Each $\phi_{i}$ can be a different limiter function, and $\psi$ is the minmod limiter function. The CFL condition insuring the positivity of the scheme (14) and (16) is

$$
\begin{aligned}
& \frac{\Delta t}{\Delta x}\left(\alpha_{x} \max _{i, q}\left|\lambda_{i}\left(\frac{\partial f}{\partial q}\right)\right|+\beta_{x} \max _{i, q} \mu_{x, i}\right) \\
& +\frac{\Delta t}{\Delta y}\left(\alpha_{y} \max _{i, q}\left|\lambda_{i}\left(\frac{\partial g}{\partial q}\right)\right|+\beta_{y} \max _{i, q} \mu_{y, i}\right) \leq \frac{1}{2} .
\end{aligned}
$$

To retain high-order accuracy in time without creating spurious oscillations, it is customary to use a TVD Runge-Kutta method [17, 18]. These Runge-Kutta methods involve a convex combination of forward Euler steps to advance the solution in time and are designed to ensure that the solution is total-variation diminishing. The second-order method reads:

$$
\begin{aligned}
& q^{(1)}=q^{n}+\Delta t \mathcal{L}\left(q^{n}\right), \\
& q^{n+1}=\frac{1}{2} q^{n}+\frac{1}{2}\left[q^{(1)}+\Delta t \mathcal{L}\left(q^{(1)}\right)\right] .
\end{aligned}
$$




\section{Simulations}

In this section we present numerical results using the positive scheme for $2 \mathrm{D}$ astrophysical jets without and with radiative cooling, and compare with WENO-LF simulations at Mach 80. (Simulations without radiative cooling are much more robust numerically, and can be done beyond Mach 1000 with both WENO-LF3 and the positive scheme, but are unphysical because radiative cooling plays a crucial role in astrophysical jets.) The positive scheme can simulate 2D jets with radiative cooling up to Mach number 270, while WENO's maximum Mach number for this problem is 135 . We first present a comparison of the positive scheme and third- and fifth-order WENO-LF with an exact 1D "jet” Riemann problem.

Computational units for the supersonic jet simulations presented in the Figures are given in Table 1 . In these units, $m_{H}=1$. The HH 1-2 astrophysical jets are modeled by the parameters given in Table 2 .

\subsection{D "Jet” Riemann Problem}

The jet Riemann problem is defined by the initial conditions (in computational units)

$$
\left[\begin{array}{l}
\rho \\
u \\
p
\end{array}\right]=\left[\begin{array}{c}
5.0 \\
30 \\
0.4127
\end{array}\right] \text { if } x \leq 0.1, \quad\left[\begin{array}{l}
\rho \\
u \\
p
\end{array}\right]=\left[\begin{array}{c}
0.5 \\
0 \\
0.4127
\end{array}\right] \text { otherwise }
$$

which model in 1D the Mach 80 jets in HH 1-2. This is a difficult Riemann problem to simulate - with two strong shock waves and a strong contact discontinuity - and serves as a good test of numerical methods which will be applied to $2 \mathrm{D}$ jet problems. To validate the positive scheme, we made comparisons (without radiative cooling) using $500 \Delta x$ in Fig. 1 with simulations using third- and fifth-order WENO-LF and with the exact solution. We used the superbee limiter for $\phi_{2}\left(\theta_{2}\right)$ and van Leer's limiter for $\phi_{i}\left(\theta_{i}\right), i=1,3$, and the parameters $\alpha=1, \beta=0.01$. The positive scheme and WENO-LF5 methods performed

Table 1 Computational units for the jets

Table 2 Parameters for the jets in $\mathrm{HH} 1-2$

\begin{tabular}{ll}
\hline Physical quantity & Basic scale \\
\hline Length & $\bar{l}=10^{11} \mathrm{~km}$ \\
Time & $\bar{t}=10^{10} \mathrm{~s}$ \\
Velocity & $\bar{u}=10 \mathrm{~km} / \mathrm{s}$ \\
Density & $\bar{\rho}=100 \mathrm{H} / \mathrm{cm}^{3}$ \\
Energy density \& pressure & $\bar{E}=\bar{P}=104.4 \mathrm{eV} / \mathrm{cm}^{3}$ \\
Temperature & $\bar{T}=1.044 \mathrm{eV} / k_{B}=12,115 \mathrm{~K}$
\end{tabular}

\begin{tabular}{ll}
\hline Jet & Ambient \\
\hline$\gamma=5 / 3$ & $\gamma=5 / 3$ \\
$\rho_{j}=500 \mathrm{H} / \mathrm{cm}^{3}$ & $\rho_{a}=50 \mathrm{H} / \mathrm{cm}^{3}$ \\
$u_{j}=300 \mathrm{~km} / \mathrm{s}$ & $u_{a}=0$ \\
$T_{j}=1000 \mathrm{~K}$ & $T_{a}=10,000 \mathrm{~K}$ \\
$c_{j}=3.8 \mathrm{~km} / \mathrm{s}$ & $c_{a}=12 \mathrm{~km} / \mathrm{s}$ \\
\hline
\end{tabular}



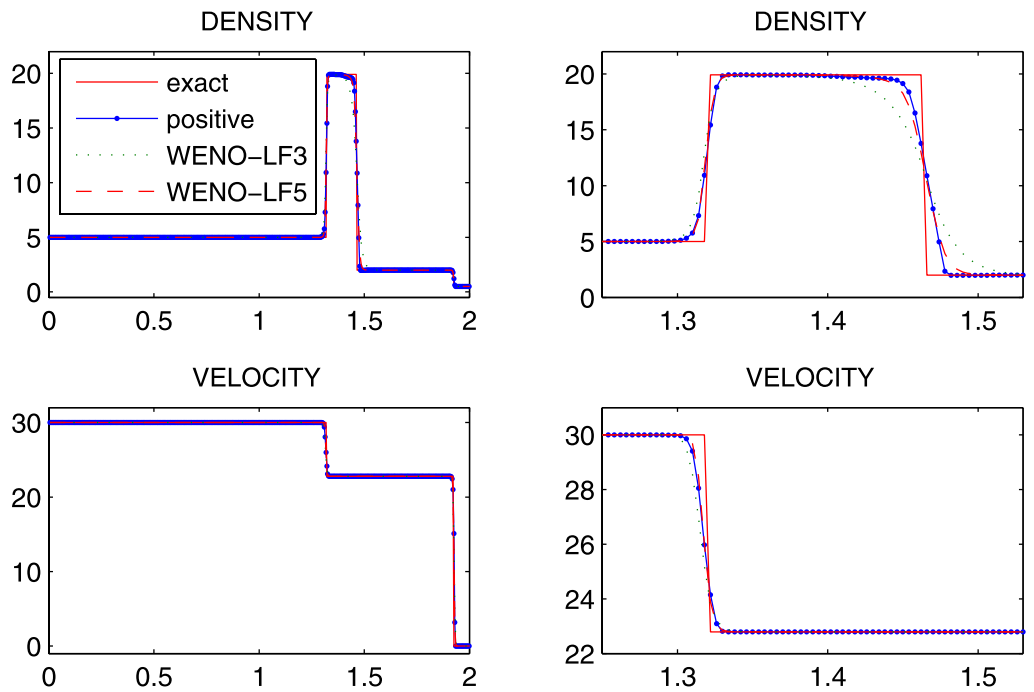

PRESSURE

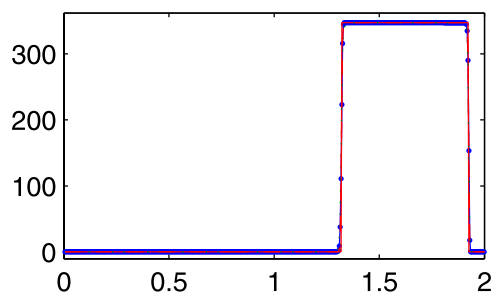

PRESSURE

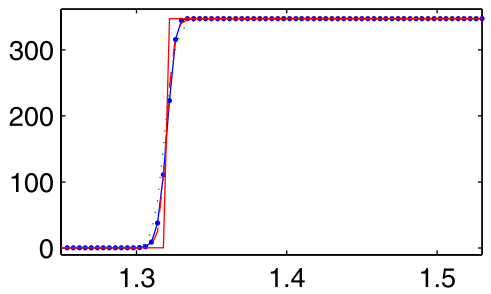

TEMPERATURE

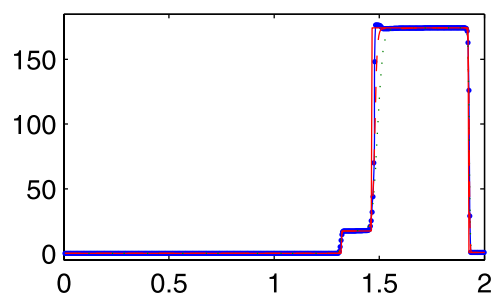

TEMPERATURE

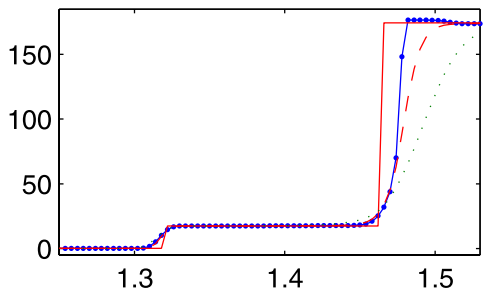

Fig. 1 Simulation of the 1D jet Riemann problem at $t_{f}=0.06: \rho\left(x, t_{f}\right), u\left(x, t_{f}\right), P\left(x, t_{f}\right)$, and $T\left(x, t_{f}\right)$. The second column of plots is a closeup of the left terminal shock and the contact

virtually identically for this Riemann problem, and both gave higher resolution of solution discontinuities than WENO-LF3. It is surprising that the second-order positive scheme is competitive with the fifth-order WENO scheme.

\subsection{D Astrophysical Jets with Radiative Cooling}

The simulations of the Mach 80 jets in $\mathrm{HH} 1-2$ were performed with the positive scheme (16) and WENO-LF3. For high Mach number jets, the WENO-LF3 method performs better than WENO-LF5 (see [2]). The jet inflow $\left(u_{j}=300 \mathrm{~km} / \mathrm{s}\right)$ is Mach 80 with respect to the soundspeed in the heavy jet gas and Mach 25 with respect to the soundspeed 


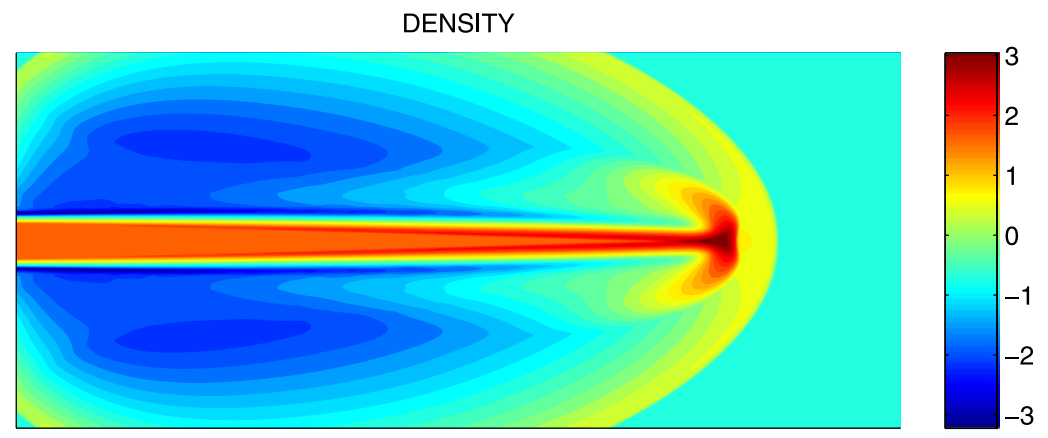

\section{PRESSURE}

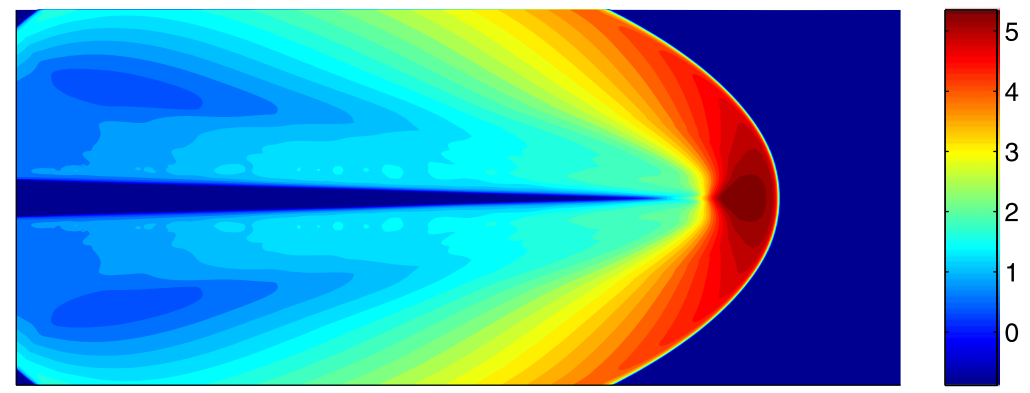

TEMPERATURE

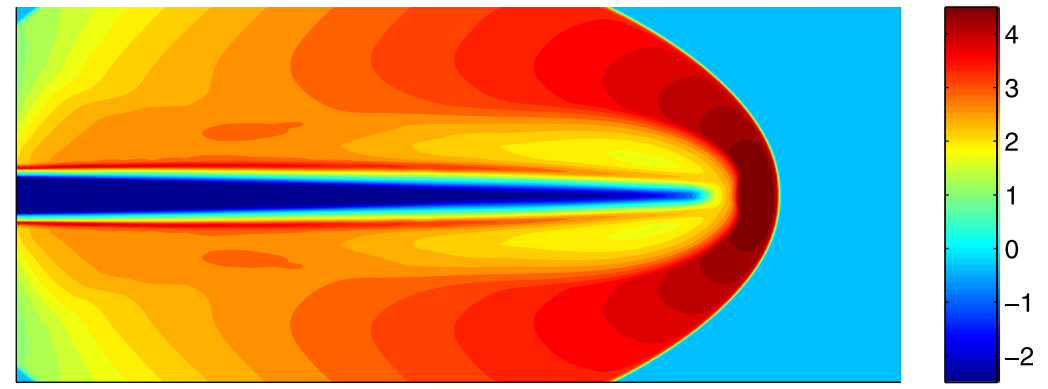

Fig. 2 Simulation of the Mach 80 jet without radiative cooling using the positive scheme $(\mathrm{CFL}=0.5)$. In all $2 \mathrm{D}$ jet figures, the physical quantities are plotted on a logarithmic scale: $\log _{10}\left\{\rho\left(x, y, t_{f}\right) / \bar{\rho}\right\}$, $\log _{10}\left\{P\left(x, y, t_{f}\right) / \bar{P}\right\}$, and $\log _{10}\left\{T\left(x, y, t_{f}\right) / \bar{T}\right\}$

in the light ambient gas. For all the $2 \mathrm{D}$ jets, the jet width is $10^{10} \mathrm{~km}$ and the jet length is approximately $1.8 \times 10^{11} \mathrm{~km}$ (the final time $t_{f}$ is chosen so that the jet has this length at $t_{f}$ ).

The initial conditions were specified by ambient values for density, pressure, and temperature, except at the left inflow boundary of the jet, where the density, pressure, and temperature were those of the jet. The ambient boundary conditions are through-flow.

Simulations of the high Mach number jets without and with radiative cooling using the positive scheme are shown in Figs. 2 and 4. We use the superbee limiter for $\phi_{i}\left(\theta_{i}\right), i=2,3$, 


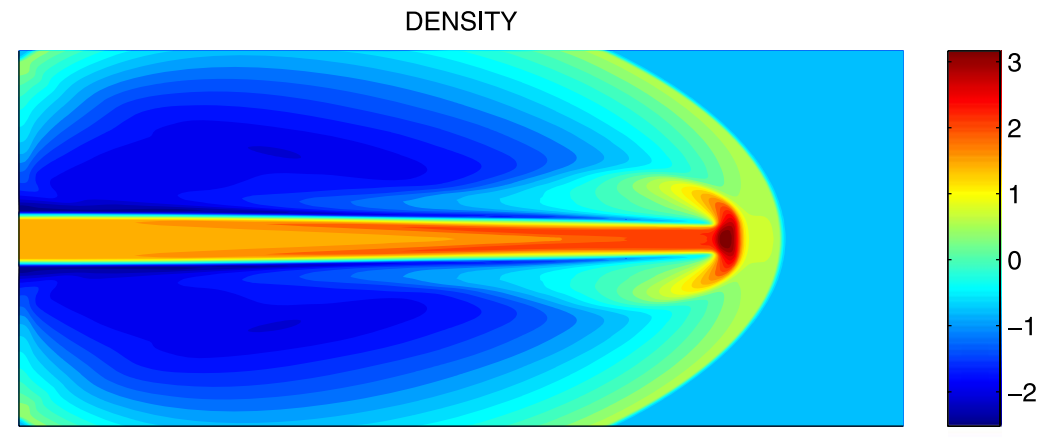

\section{PRESSURE}

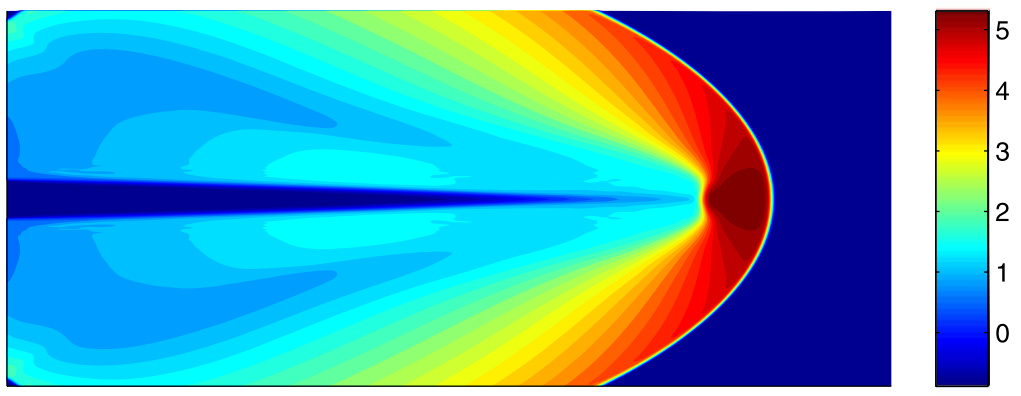

TEMPERATURE

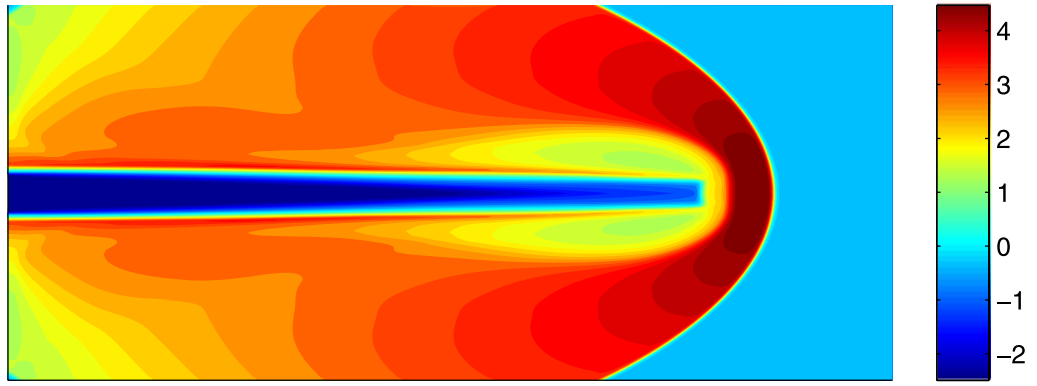

Fig. 3 Simulation of the Mach 80 jet without radiative cooling using third-order WENO-LF $(\mathrm{CFL}=0.45)$

and van Leer's limiter for $\phi_{i}\left(\theta_{i}\right), i=1,4$, and the parameters $\alpha_{x}=\alpha_{y}=1, \beta_{x}=2$, and $\beta_{y}=5$, with $500 \Delta x$ and $250 \Delta y$. Figures 3 and 5 demonstrate that the positive scheme and WENO-LF3 have nearly identical resolution (we chose the maximum CFL factor for each method that gave good results: $\mathrm{CFL}=0.5$ for the positive scheme and $\mathrm{CFL}=0.45$ for WENO-LF3). Small differences in the simulations are apparent for the two methods, but the images are so close it is impossible to say which method gives better results at Mach 80 . Note that contours are very sensitive to small changes in solution values, and that contours for the same jet problem will differ because the computed ranges of physical quantities for the two methods differ slightly. 

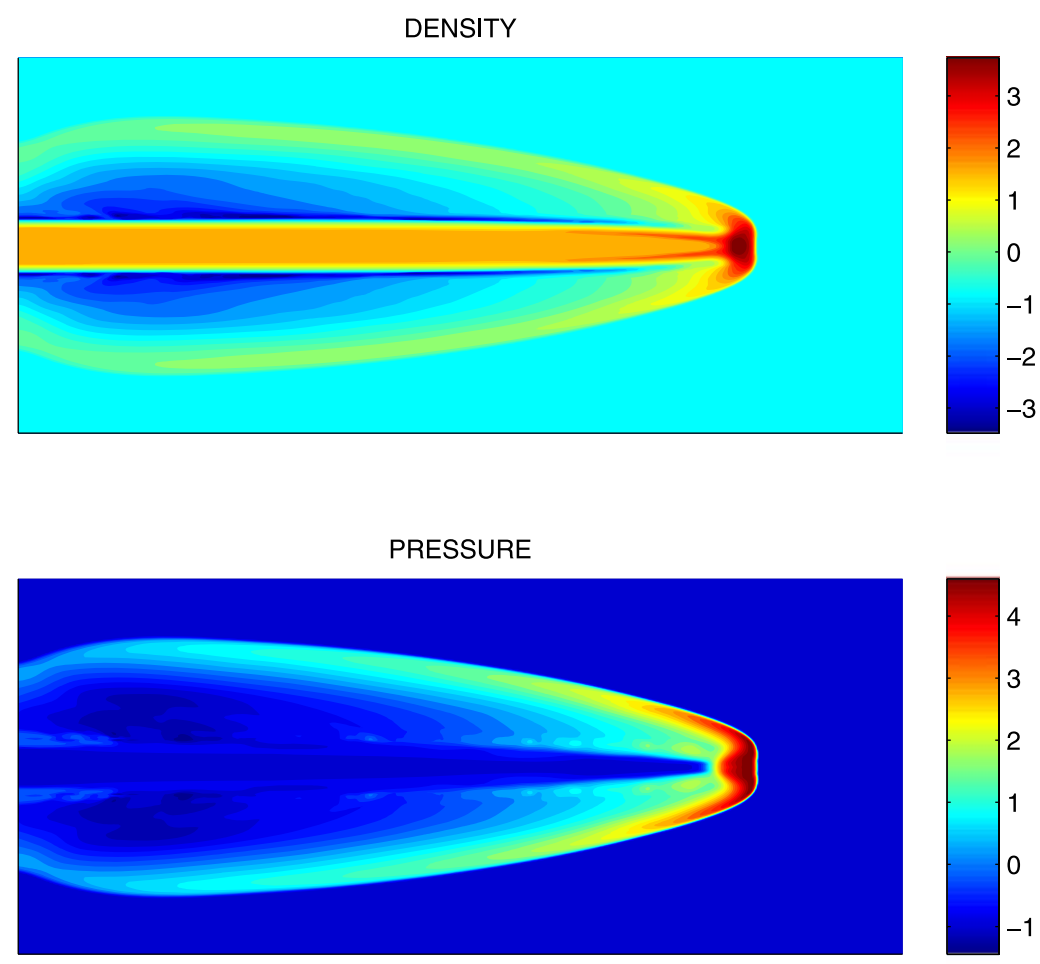

TEMPERATURE

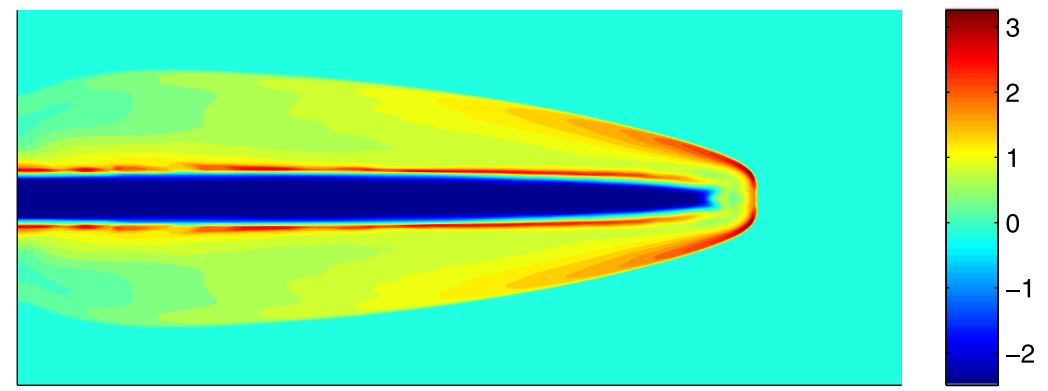

Fig. 4 Simulation of the Mach 80 jet with radiative cooling using the positive scheme $(\mathrm{CFL}=0.5)$

The simulations accurately reproduce the morphology and physics of astrophysical jets, including the bow shock ahead of the jet, the terminal Mach disk shock just inside the tip of the jet, and the Kelvin-Helmholtz rollup of the jet tip. Note the differences between the jets without and with radiative cooling. The jet with radiative cooling has a much higher density contrast near the jet tip (since as the shocked, heated gas cools radiatively, it compresses) and a much thiner bow shock, reduced Kelvin-Helmholtz rollup of the jet tip, and a lower average temperature.

Finally in Fig. 6, we show that the positive scheme can simulate astrophysical jets with radiative cooling up to Mach $270\left(u_{j}=1000 \mathrm{~km} / \mathrm{s}\right)$ with respect to the heavy jet gas (Mach 

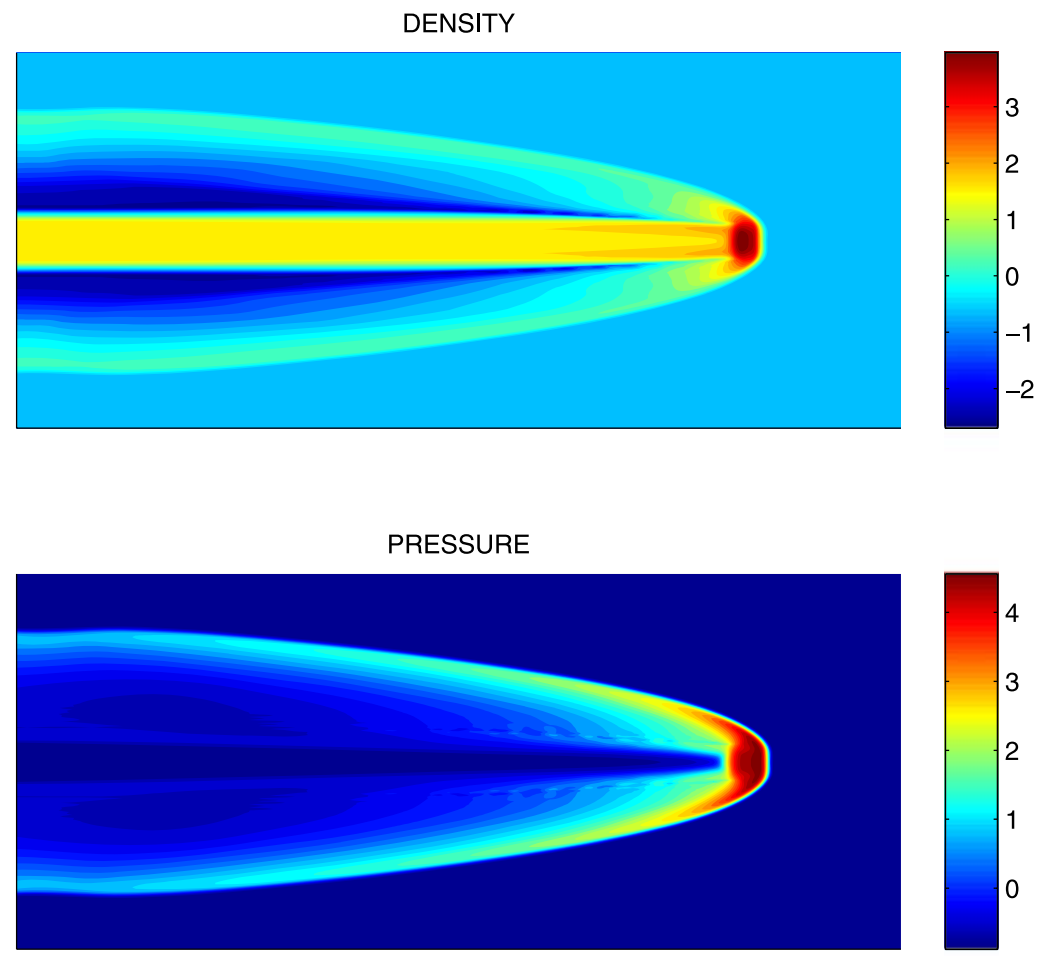

TEMPERATURE

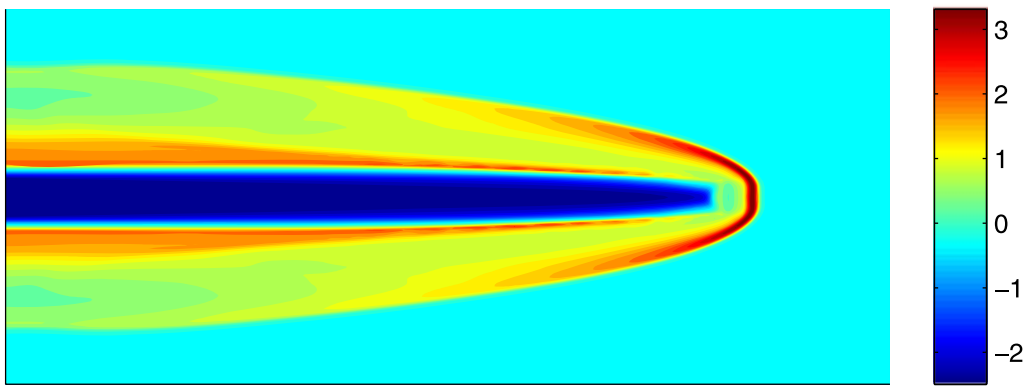

Fig. 5 Simulation of the Mach 80 jet with radiative cooling using third-order WENO-LF $(\mathrm{CFL}=0.45)$

85 with respect to the light ambient gas), a factor of two times higher than the maximum Mach number attained with the WENO-LF3 scheme. Note that the Mach 270 jet with radiative cooling has similar structures to the Mach 80 jet with radiative cooling, which was verified against Hubble images of HH 1-2, except that the Mach 270 jet is more ballistic, has more dramatic heating and cooling, and has stronger radiative shocks, as expected.

It is plausible [19] that the positive scheme is able to simulate astrophysical jets with radiative cooling at much higher Mach numbers than WENO-LF because the positive scheme uses a limiter which clips extrema (formally reducing it to first-order accuracy near shocks), 

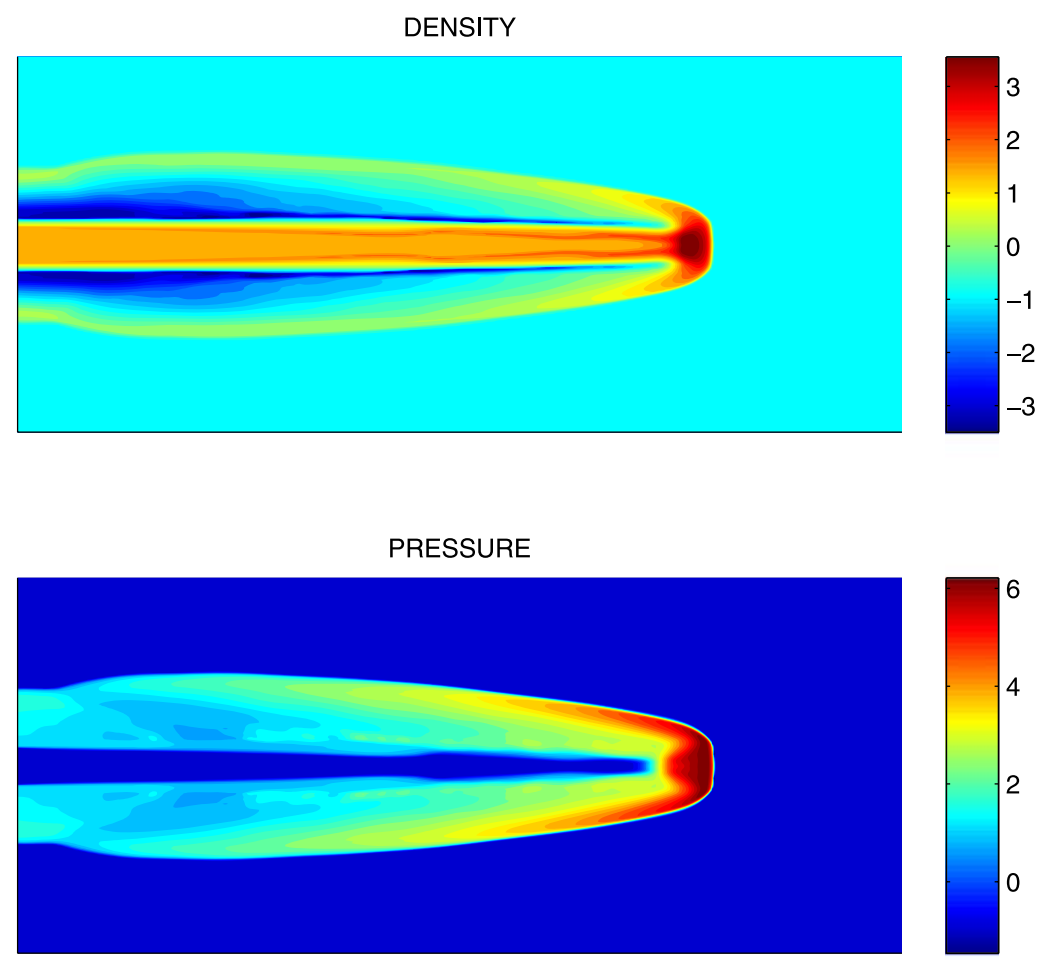

TEMPERATURE

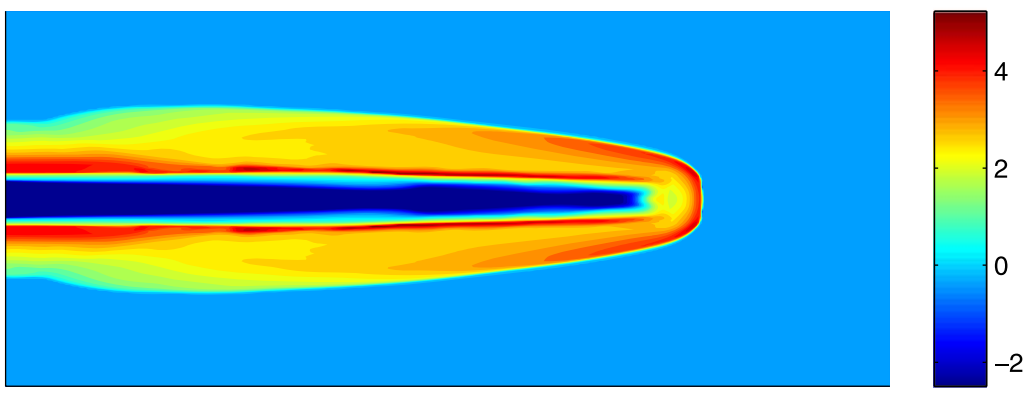

Fig. 6 Simulation of the Mach 270 jet with radiative cooling using the positive scheme $(\mathrm{CFL}=0.5)$

which makes it more stable against generating negative pressures or densities near strong shocks - the major source of instability in these jet computations.

\section{Conclusion}

Hubble, Spitzer (infra-red), and Chandra (X-ray) space telescope images have revealed a new wealth of detail in high Mach number gas flows and shock wave patterns involving astrophysical jets, colliding interstellar winds of particles, colliding shock waves, shock wave- 
nebula interactions, the interaction of strong shocks with winds, and the interaction of shock waves with proto-planetary disks around young stars. Simulations at high Mach number and with radiative cooling are essential in understanding the density contrasts, morphologies, and strong radiative shocks in these astrophysical processes.

Hyperbolic numerical methods eventually produce negative pressures or densities and fail as the maximum Mach number of the jet problem is increased. With radiative cooling, stronger shocks are produced than without cooling, so simulations with cooling fail at lower Mach numbers than without cooling. However no negative pressures or densities are generated in the simulations presented in the figures, even at much later times than shown in the figures.

The positive scheme outlined here has proven to be very robust for simulating astrophysical jets at extremely high Mach numbers with radiative cooling. It should be an ideal method for investigating the wide variety of high Mach number radiative flows which occur in astrophysical images.

\section{References}

1. Hester, J.J., Stapelfeldt, K.R., Scowen, P.A.: Hubble space telescope wide field planetary camera 2 observations of HH 1-2. Astron. J. 116, 372-395 (1998)

2. Ha, Y., Gardner, C.L., Gelb, A., Shu, C.-W.: Numerical simulation of high Mach number astrophysical jets with radiative cooling. J. Sci. Comput. 24, 29-44 (2005)

3. Gardner, C.L., Ha, Y., Hester, J.J., Krist, J.E., Shu, C.-W., Stapelfeldt, K.R.: Numerical simulation of high Mach number astrophysical jets. In: Analysis, Modeling and Computation of Hyperbolic PDE and Multiphase Flow. SUNY, Stony Brook (2005)

4. Liu, X.-D., Lax, P.D.: Positive schemes for solving multi-dimensional hyperbolic systems of conservation laws. J. Comput. Fluid Dyn. 5, 133-156 (1996)

5. Lax, P.D., Liu, X.-D.: Solution of two-dimensional Riemann problems of gas dynamics by positive schemes. SIAM J. Sci. Comput. 19, 319-340 (1998)

6. Jiang, G.-S., Shu, C.-W.: Efficient implementation of weighted ENO schemes. J. Comput. Phys. 126, 202-228 (1996)

7. Shu, C.-W.: High order ENO and WENO schemes for computational fluid dynamics. In: High-Order Methods for Computational Physics. Lecture Notes in Computational Science and Engineering, vol. 9, pp. 439-582. Springer, New York (1999)

8. LeVeque, R.J.: CLAWPACK website. http://www.amath.washington.edu/ claw/

9. LeVeque, R.J.: Finite Volume Methods for Hyperbolic Problems. Cambridge University Press, Cambridge (2002)

10. Schmutzler, T., Tscharnuter, W.M.: Effective radiative cooling in optically thin plasmas. Astron. Astrophys. 273, 318-330 (1993)

11. Lax, P.D., Wendroff, B.: Systems of conservation laws. Commun. Pure Appl. Math. 13, 217 (1960)

12. Borice, J.P., Book, D.L.: Flux corrected transport I, SHASTA, a fluid transport algorithm that works. J. Comput. Phys. 11, 38-69 (1973)

13. Harten, A., Zwas, G.: Self-adjusting hybrid schemes for shock computations. J. Comput. Phys. 9, 568-583 (1973)

14. Roe, P.L.: Approximate Riemann solvers, parameter vectors, and difference schemes. J. Comput. Phys. 43, 357-372 (1981)

15. Harten, A.: On a class of high resolution total-variation-stable finite-difference schemes. SIAM J. Numer. Anal. 21, 1-23 (1984)

16. Sweby, P.K.: High resolution schemes using flux limiters for hyperbolic conservation laws. SIAM J. Numer. Anal. 21, 995-1011 (1984)

17. Shu, C.-W.: Total-variation-diminishing time discretizations. SIAM J. Sci. Stat. Comput. 9, 1073-1084 (1988)

18. Shu, C.-W., Osher, S.: Efficient implementation of essentially non-oscillatory shock capturing schemes II. J. Comput. Phys. 83, 32-78 (1989)

19. Shu, C.-W.: Private communication 